\title{
Das Digitale-Versorgung-Gesetz - Versorgungsoptimierung oder Wirtschaftsförderung?
}

MELANIE SCHNEE, STEFAN GREß

Dr. Melanie Schnee ist Lehrkraft für besondere Aufgaben am Fachbereich Pflege und Gesundheit an der Hochschule Fulda

Prof. Dr. Stefan Greß ist Hochschullehrer für Versorgungsforschung und Gesundheitsökonomie am Fachbereich Pflege und Gesundheit an der Hochschule Fulda

\author{
Das Digitale-Versorgung-Gesetz ist beschlossene \\ Sache. Digitale Gesundheitsanwendungen sollen \\ schon bald die Gesundheitsversorgung verbessern. \\ In der öffentlichen Debatte des Gesetzes ging es \\ vor allem um Fragen des Datenschutzes. Der Beitrag \\ überprüft die vom Gesetzgeber vorgesehenen \\ Steuerungsmechanismen: Wird das DVG tatsächlich \\ eine Verbesserung der Versorgung bringen oder \\ handelt es sich vielmehr bei dem Gesetz um verdeckte \\ Wirtschaftsförderung auf Kosten der Beitragszahler?
}

\section{Einleitung}

Dem deutschen Gesundheitssystem wird bei der Digitalisierung im internationalen Vergleich Aufholbedarf attestiert (Bertelsmann Stiftung 2018). Daher wird die primäre Intention des Digitale Versorgung-Gesetzes (DVG) - digitale Innovationen schneller in die Versorgung zu bringen - geradezu einhellig von allen Akteuren begrüßt. Auch wenn in der öffentlichen Wahrnehmung des DVG eher Fragen des Datenschutzes und der Nutzung von Gesundheitsdaten für Forschungszwecke diskutiert werden, lohnt ein genauer Blick auf die Steuerungsmechanismen des Gesetzes. Der Hauptschuldige für die Trägheit des Gesundheitswesens bei der Digitalisierung scheint jedenfalls ausgemacht. Dies ist für den Gesetzgeber offensichtlich die gemeinsame Selbstverwaltung mit ihren Partikularinteressen, die bereits bei der Einführung der elektronischen Gesundheitskarte mehr Bremsklotz als Motor war. Die gemeinsame Selbstverwaltung wird vom DVG weitgehend ausgehebelt. Damit sollen Entscheidungsprozesse beschleunigt und die Versorgung durch innovative digitale Anwendungen verbessert werden. Die absehbaren Wirkungen und Nebenwirkungen dieses Vorgehens analysieren wir in diesem Beitrag.

\section{Notwendige Rahmenbedingungen für erfolgreiche Digitalisierung}

Digitalisierung ist kein Prozess, der losgelöst von anderen strukturellen Fragen der Versorgungsorganisation vorangetrieben werden kann. Digitalisierung ist nur im Zusammenspiel mit einer Vernetzung und damit Integration mit allen an der Gesundheitsversorgung beteiligten Akteuren sinnvoll. Die Integration des Gesundheitswesens, die bisher oftmals nur als vertikale Integration zwischen primärer, sekundärer und tertiärer Versorgung diskutiert wurde, muss nun umfassender gedacht werden und bezieht ein digitales Schnittstellenmanagement, die Nutzung von Daten zur Generierung klinischer Expertise und den Patienten als Co-Produzenten von Informationen mit ein (Amelung et al. 2017). Eine gelun- 
gene Digitalisierung erzwingt geradezu eine Integration von Versorgungsketten von der Prävention und Gesundheitsförderung über Diagnostik und Therapie bis zur Rehabilitation einschließlich der Stärkung des Patienten in seinem Gesundheits- und Krankheitsmanagement (Meister 2019: 12)

Die WHO mahnt in ihren Empfehlungen ebenfalls ein strategisches Vorgehen bei der Digitalisierung von Gesundheitssystemen an: Digitalisierung ist danach allerdings kein Selbstzweck, sondern muss primär den nationalen Gesundheitszielen dienen (WHO 2019: 81). Kern der Digitalisierung ist die Vernetzung der Gesundheitsberufe, Gesundheitseinrichtungen und Kunden (WHO 2019: 83), die durch einen angemessenen rechtlichen Rahmen, geeignete Finanzierungsformen sowie Weiterbildungsangebote für die Gesundheitsberufe und die potentiellen Nutzer unterstützt werden müssen.

Die zögerliche Digitalisierung im deutschen Gesundheitssystem ist in diesem Sinne eine „Integrierte Versorgung 2.0“. Die Probleme bei der Digitalisierung sind analog zur schleppenden Integration von klassischen Versorgungsstrukturen zu sehen - beiden Themen fehlte bisher die Vision, die in eine politische Strategie übersetzt wird. Das DVG versucht, vor allem über eine beschleunigte Einführung von digitalen Gesundheitsanwendungen, Druck auf die Organisation der Versorgung auszuüben. Der Gesetzgeber bewertet das DVG daher auch nur als einen weiteren wichtigen Schritt „im Rahmen eines iterativen Gesamtprozesses“ (Deutscher Bundestag 2019a: 2). Die strukturelle Voraussetzung - die digitale Vernetzung der betroffenen Akteure - wird durch die Verschiebung der Einführung der elektronischen Patientenakte auf einen späteren Zeitpunkt allerdings nicht geschaffen. Der derzeitige „Wildwuchs an kleinteiligen Systemen, Programmen und Angeboten“ (Amelung/Ex 2019: 17) wird nun durch weitere Angebote ergänzt.

\section{Regulierungsrahmen für digitale Gesundheitsanwendungen}

Kernpunkt des Gesetzes ist ein Regulierungsrahmen zur Aufnahme digitaler Gesundheitsanwendungen in die Regelversorgung. Dazu hat der Gesetzgeber Mechanismen entwickelt, die sowohl die
Erstattungsfähigkeit dieser Anwendungen als auch die Preisfindung normieren. Gesetzliche Krankenkassen erhalten außerdem die Möglichkeit, in die Weiterentwicklung digitaler Innovationen zu investieren. Letztlich sollen Versicherte bei dem Erwerb digitaler Gesundheitskompetenzen gefördert werden.

Nach $\mathbb{3} 33$ a SGB V haben Versicherte zukünftig einen leistungsrechtlichen Anspruch auf die Versorgung mit „Medizinprodukten niedriger Risikoklasse, deren Hauptfunktion wesentlich auf digitalen Technologien beruht und die dazu bestimmt sind, bei den Versicherten oder in der Versorgung durch Leistungserbringer die Erkennung, Überwachung, Behandlung oder Linderung von Krankheiten oder die Erkennung, Behandlung, Linderung oder Kompensierung von Verletzungen oder Behinderungen zu unterstützen." ${ }^{1}$ Versicherte haben nur auf solche digitalen Gesundheitsanwendungen einen Anspruch, die vom Bundesinstitut für Arzneimittel und Medizinprodukte (BfArM) in das Verzeichnis digitaler Gesundheitsanwendungen nach $\$ 139$ e SGV aufgenommen wurden. Zudem müssen die Anwendungen entweder vom behandelnden Arzt verordnet oder mit Genehmigung der Krankenkassen angewendet werden. Für eine Genehmigung der Krankenkasse müssen die Versicherten zudem das Vorliegen einer medizinischen Indikation nachweisen.

Voraussetzung für die Erstattungsfähigkeit von digitalen Gesundheitsanwendungen ist demzufolge die Aufnahme der Anwendung in das beim BfArM geführte Verzeichnis. Die Aufnahme erfolgt auf Antrag des Herstellers. Nach $\$ 139$ e SGB V Abs. 2 müssen die Hersteller in diesem Antrag erstens nachweisen, dass die digitale Anwendung den Anforderungen an Sicherheit, Funktionstauglichkeit und Qualität des Medizinproduktes entspricht. Zweitens müssen Anforderungen an Datenschutz und Datensicherheit gewährleistet sein. Drittens muss die digitale Gesundheitsanwendung „positive Versorgungseffekte“ aufweisen. Letztere eher unbestimmte Formulierung im Gesetzentwurf der Bundesregierung wurde im Laufe des Gesetzgebungsverfahrens weiter operationalisiert. Ein positiver Versorgungseffekt ist demnach entweder ein medizinischer Nutzen oder eine patientenrelevante Struktur- und Verfahrensverbesserung in der Versorgung. In der Begründung des Gesetzesentwurfs war noch davon die Rede, dass unter positiven Versorgungseffekten ein medizinischer Nutzen „im engeren Sinn einer therapeutischen Verbesserung durch positive Beeinflussung patientenrelevanter Endpunkte wie der Lebensqualität“ $\mathrm{zu}$ verstehen ist (Deutscher Bundestag 2019b: 59).

Falls der Hersteller einen Nachweis über positive Versorgungseffekte nicht führen kann, kann das BfArM die Anwendung auf Antrag für einen Zeitraum von bis zu zwölf Monaten zur Erprobung in das Verzeichnis aufnehmen. Eine Verlängerung des Erprobungszeitraums um weitere zwölf Monate ist möglich, wenn eine hohe Wahrscheinlichkeit eines positiven Versorgungseffektes besteht. Sollte der Nutzennachweis ausbleiben, ist die digitale Gesundheitsanwendung aus dem Verzeichnis zu streichen. Eine wiederholte vorläufige Aufnahme in das Verzeichnis zur Erprobung soll ausgeschlossen werden ( $\$ 139$ e SGB V Abs. 4).

Beim Nachweis eines positiven Versorgungseffekts sind zwar die Grundsätze der evidenzbasierten Medizin zu beachten ( $\$$ 139e SGB V Abs. 9). Der Nachweis des Nutzens kann laut Gesetzesbegründung allerdings durch „Fallberichte, Expertenmeinungen, Anwendungsbeobachtungen, Studien oder sonstige valide Erkenntnisse“ erfolgen (Deutscher Bundestag 2019b: 59). Diese eher niedrigen Anforderungen an die Evidenz über den Nutzen werden mit dem geringen Risikopotenzial für den Patienten, den vergleichswiese niedrigen Kosten und dem praktischen Mehrwert digitaler Gesundheitsanwendungen begründet. In der weiteren Begründung wird auf die integrierende Funktion von digitalen Gesundheitsanwendungen hingewiesen, denn auch „Verfahrens- und Strukturverbesserungen in der gesundheitlichen Versorgung, wie beispielsweise eine sachgerechte Inanspruchnahme ärztlicher und anderer Leistungserbringer, eine bessere Koordinierung der Versorgungsabläufe, die Förderung der Patienteninformation und Patientensouveränität, die Bewäl-

1 Bei Erstellung dieses Beitrags war das DVG im Bundesanzeiger noch nicht veröffentlicht. Sämtliche Zitate gesetzlicher Regelungen im SGB V beziehen sich daher auf die im Bundestag am 7. November 2019 beschlossene Empfehlung des Ausschusses für Gesundheit vom 6. November 2019 (Deutscher Bundestag 2019a). 
tigung krankheitsbedingter praktischer Schwierigkeiten " können die Erstattung durch die GKV rechtfertigen (Deutscher Bundestag 2019b: 59). Eine gesundheitsökonomische Evaluation digitaler Gesundheitsanwendungen wird an keiner Stelle des Gesetzes eingefordert. Näheres zu den Anforderungen an das Evaluationskonzept zum Nachweis positiver Versorgungseffekte soll die Bundesregierung in einer Rechtsverordnung regeln. In der Gesetzesbegründung wird zudem auf das Evidence Standards Framework for Digital Health Technologies des NICE verwiesen (Deutscher Bundestag 2019a: 96).

Das Verfahren für die Festlegung der Erstattungspreise für digitale Gesundheitsanwendungen normiert der Gesetzgeber in $\$ 134$ SGB neu. Insge-

\section{Die Zulassung von digitalen Gesundheitsanwendungen über das BfArM wird zu neuen Schnittstellenproblemen führen.}

im ersten Jahr der Markteinführung erbracht wurde. Es sind demnach Vergütungsabschläge für solche Produkte vorzusehen, zu denen entsprechende Nachweise noch nicht vorliegen.

Den Regulierungsrahmen für die Erstattung digitaler Gesundheitsanwendungen ergänzt der Gesetzgeber durch zwei weitere Maßnahmen - die Förderung der digitalen Gesundheitskompetenz der Patienten und die optionale Förderung der Entwicklung von digitalen Versorgungsinnovationen durch die Krankenkassen.

Nach $\ 20$ k SGB V haben die Krankassen in der Satzung Leistungen zur Förderung des selbstbestimmten gesundheitsorientierten Einsatzes digitaler oder telemedizinischer Anwendungen und Verfahren durch die Versicherten vorzusehen. Die Leistungen sollen dazu dienen, die für die Nutzung digitaler oder telemedizinischer Anwendungen und Verfahren erforderlichen Kompetenzen zu vermitteln. Die Vermittlung von digitalen Gesundheitskompetenzen soll einen Beitrag zur Verminde-

samt erinnert dieses Verfahren stark an die Regelungen im Arzneimittelmarktneuordnungsgesetz (AMNOG). Auch hier soll der Spitzenverband Bund der Krankenkassen mit den Herstellern digitaler Gesundheitsanwendungen Erstattungspreise für digitale Gesundheitsanwendungen verhandeln. Gegenstand der Verhandlungen sollen auch „erfolgsabhängige Preisbestandteile“ sein (\$S 134 SGB V Abs. 1). Bei Nichteinigung erfolgt die Preisfestsetzung durch eine Schiedsstelle. Die Erstattungspreise gelten - eine weitere Parallele zum AMNOG-Verfahren - erst ein Jahr nach Aufnahme der jeweiligen digitalen Gesundheitsanwendung in das BfArM-Verzeichnis.

Erst im Laufe des Gesetzgebungsverfahrens wurde diese - zumindest aus der Sicht der Hersteller digitaler Gesundheitsanwendungen - außerordentlich großzügige Regelung modifiziert. In $\mathbb{} 134$ SGB V Abs. 5 ist daher normiert, dass Hersteller und Krankenkassen kollektivvertraglich vereinbarte Erstattungshöchstbeträge für Gruppen vergleichbarer digitaler Gesundheitsanwendungen auch in Abhängigkeit davon vereinbaren müssen, ob ein Nachweis positiver Versorgungseffekte bereits rung der Ungleichheit von Gesundheitschancen leisten (Deutscher Bundestag 2019a: 90).

Den Krankenkassen wird zudem durch die Neureglung in $\$ 68$ a SGB V die Möglichkeit eröffnet, die Entwicklung digitaler Innovationen zu fördern. Die Förderung muss möglichst bedarfsgerecht und zielgerichtet sein sowie zur Verbesserung der Versorgungsqualität und Versorgungseffizienz, zur Behebung von Versorgungsdefiziten und zur verbesserten Patientenorientierung in der Versorgung beitragen ( $\mathbb{S} 68$ a SGB $\mathrm{V}$ Abs. 1). Unter digitalen Innovationen sind gemäß Abs. 2 digitale Medizinprodukte, telemedizinische Verfahren oder IT-gestützte Verfahren in der Versorgung zu verstehen. Die Förderung erfolgt entweder durch eine fachlich-inhaltliche Kooperation oder durch einen Erwerb von Anteilen an Investmentvermögen, soweit sie mit einer fachlich-inhaltlichen Kooperation verbunden wird. Nach $\mathbb{}$ 263a Abs. 1 können Krankenkassen bis zu zwei Prozent ihrer Finanzreserven für diesen Zweck verwenden. Diese sind so anzulegen, dass die Kapitalbindungsdauer zehn Jahre nicht überschreitet, ein „Verlust ausgeschlossen erscheint und ein angemessener Ertrag erzielt" wird (\$ 263a SGB V Abs. 2).

\section{Bewertung des Regulierungsrahmens}

Der oben beschriebene Regulierungsrahmen im DVG lässt sich aus unserer Sicht insbesondere im Hinblick auf drei Dimensionen kritisieren. Erstens kann von einer Digitalisierungsstrategie der Bundesregierung keine Rede sein. Die schon angesprochenen „iterativen Maßnahmen" lassen eher auf Aktionismus und Symbolpolitik schließen. Zweitens hebeln die Regelungen zur Erstattungsfähigkeit von digitalen Gesundheitsanwendungen die Institutionen der gemeinsamen Selbstverwaltung weitgehend aus und legen einen ausgesprochen niedrigen Evidenzstandard an. Drittens bergen die Regelungen zur Preisregulierung erheblicher Ausgabenrisiken für die Beitragszahler in der gesetzlichen Krankenversicherung.

\subsection{Iterative Maßnahmen statt Digitalisierungsstrategie}

Die oben angesprochenen Voraussetzungen für eine erfolgreiche Digitalisierungsstrategie - insbesondere Vernetzung, Integration und Orientierung an Gesundheitszielen - werden durch das DVG nicht geschaffen, sondern offensichtlich vom Gesetzgeber vorausgesetzt. Die weitgehend ungesteuerte Finanzierung von digitalen Gesundheitsanwendungen wird nach unserer Prognose diese Vernetzung und Integration nicht befördern, sondern eher $\mathrm{zu}$ neuen Schnittstellenproblemen führen. Dies gilt insbesondere für die Beziehung zwischen Ärzten und Krankenkassen. Schließlich gehöre es nach der Gesetzesbegründung nun zum Versorgungsauftrag der Vertragsärzte, die Anwendungsergebnisse auch der digitalen $\mathrm{Ge}$ sundheitsanwendungen in die ärztliche Behandlung einzubeziehen, die sie nicht selbst verordnet haben (Deutscher Bundestag 2019b: 44).

Es ist zudem widersprüchlich, dass zwar die Ziele der Verbesserung der Versorgungsqualität und Versorgungseffizienz genannt werden, der Gesetzentwurf jedoch keine zwingende Nutzenbewertung oder noch besser Kosten-NutzenBewertung bei der Verwendung von Mitteln vorsieht, die streng genommen 
für die Versorgung im Krankheitsfall gedacht sind. Diese Form der Steuerung ist somit ein Rückfall in die Zeiten der Anschubfinanzierung der Integrierten Versorgung: hier waren die Evaluation der Verträge und demnach auch die $\mathrm{Pu}-$ blikation der Ergebnisse freiwillig. In einem Wettbewerb der Ideen haben die Krankenkassen aus ihrer Perspektive heraus keinen Anreiz, der Konkurrenz Erfolge oder auch Misserfolge zu dokumentieren, da der Mitbewerber aus den Evaluationsergebnissen lernen und damit wiederum einen Wettbewerbsvorteil erzielen könnte.

\subsection{Systembruch bei der Erstattungsfähigkeit digitaler Gesundheitsanwendungen}

An der vorgesehenen Regelung ist insbesondere zu bemängeln, dass die digitalen Gesundheitsanwendungen einen privilegierten Zugangsweg erhalten, während Arzneimittel und andere innovative medizinische Technologien ein eher langwieriges Verfahren beim Gemeinsamen Bundesausschuss (G-BA) durchlaufen müssen. Den G-BA bei diesem Verfahren auszuschließen, ist sicherlich dem Wunsch nach schneller Einführung von digitalen Gesundheitsanwendungen geschuldet. Schließlich soll gemäß Gesetzesbegründung ein „Fast Track“ (Deutscher Bundestag 2019b: 84) für digitale Gesundheitstechnologien geschaffen werden. Insgesamt folgt der Gesetzgeber mit dem beschriebenen Regulierungsrahmen dem Zentralisierungspfad der vergangenen Jahre (Greß/ Heinemann 2018). Dies wird insbesondere dadurch deutlich, dass keine Institution der gemeinsamen Selbstverwaltung, sondern eine an sich fachfremde Bundesbehörde mit der Erstellung des Registers von erstattungsfähigen digitalen Gesundheitsanwendungen beauftragt wird (Egger 2019).

Den noch weitaus schwerwiegenderen Systembruch stellen aus unserer Sicht die geringen Anforderungen an die Erstattungsfähigkeit digitaler Gesundheitsanwendungen dar. Der Begriff der positiven Versorgungseffekte ist weitgehend unbestimmt und lässt der Bundesregierung bei der Erstellung der entsprechenden Verordnung große Spielräume. Mit den geringen Anforderungen an die vorzulegende Evidenz wird suggeriert, dass Gesundheits-Apps genau die Probleme lösen, die die Gesundheitspolitik seit Jahren nicht lösen konnte. Es wird dabei ebenfalls unterschlagen, dass es genau für diese Prozess- und Strukturverbesserungen einer völligen Vernetzung der aller Leistungserbringer sowie einer digitalen Plattform zum Austausch von Patientendaten - etwa in Form einer elektronischen Patientenakte - bedarf, die durch die aktuellen gesetzlichen Regelungen jedenfalls noch nicht realisiert werden können. Die WHO warnt in ihren Leitlinien zur Digitalisierung vor einer Überschätzung der Wirkung digitaler Anwendungen, da oftmals andere Faktoren wie parallele Therapien ebenfalls eine Rolle bei der Verbesserung des Gesundheitszustands spielen können. Für eine fundierte Nutzenbewertung wird zudem vorgeschlagen, dass sowohl die technologische Funktionalität als auch das Potential zur Verhaltensänderung

\section{Mit den geringen Anforderungen an die Evidenz wird suggeriert, dass Gesundheits-Apps die Probleme lösen, die die Gesundheitspolitik seit Jahren nicht lösen konnte.}

\subsection{Hohe Ausgabenrisiken für die GKV}

Der Regulierungsrahmen für die Festsetzung von Preisen digitaler Gesundheitsanwendungen orientiert sich weitgehend an der AMNOG-Systematik.

Auch bei Arzneimitteln ist zu kritisieren, dass Hersteller im ersten Jahr nach der Markteinführung die Preise frei festsetzen können, die dann als Grundlage für die Preisverhandlungen mit dem Spitzenverband Bund der Krankenkassen dienen. Bei Arzneimitteln war offensichtlich der von den Herstellern immer wieder angeführte hohe Aufwand für

und die Auseinandersetzung des Nutzers mit den Inhalten evaluiert werden (WHO 2019: 86).

Auch der Verzicht auf gesundheitsökonomische Evaluationen ist kritisch zu bewerten. Dabei kann es durchaus sinnvoll sein, das Kosten-Nutzen-Verhältnis digitaler Gesundheitsanwendungen in den Blick zu nehmen. Die WHO schlägt in ihren Leitlinien vor, dass die Kosten von E-Health-Produkten über einen längeren Zeitraum verfolgt werden sollten, um auch den Unterhalt der Anwendung (etwa im Hinblick auf Updates) und die Unterstützung der Nutzer bei Problemen angemessen zu erfassen (WHO 2019: 87).

Gerade bei Produkten, die in die Therapie eingreifen, ist auch die begleitende Unterstützung durch Leistungserbringer mit einzurechnen. Die Hersteller von digitalen Gesundheitsanwendungen werden schon aus reinem Selbstschutz zum Ausschluss von Haftungsrisiken die Algorithmen der Apps derart gestalten, dass bei jeglichen Unregelmäßigkeiten
Forschung und Entwicklung Grund für dieses Zugeständnis des Gesetzgebers. gitaler Gesundheitsanwendungen in Forschung und Entwicklung investieren. Ein vergleichbarer Aufwand wie bei innovativen verschreibungspflichtigen Arzneimitteln ist jedoch nicht zu erkennen. Insofern ist es aus unserer Sicht nicht zu rechtfertigen, warum Hersteller digitaler Gesundheitsanwendungen zumindest im ersten Jahr nach der Erstattungsfähigkeit die Preise frei zu Lasten der Beitragszahler in der gesetzlichen Krankenversicherung festlegen können. Die deutliche Kritik an diesen Regelungen hat erst im Laufe des Gesetzgebungsverfahrens dazu geführt, dass Preisabschläge für solche Anwendungen vorzusehen sind, die noch keine Belege für den praktischen Mehrwert vorgelegt haben. Nichtsdestoweniger scheint aus Sicht des Gesetzgebers das Risiko von Mondpreisen für digitale Anwendungen in Kombination mit äußerst bescheidenen Anforderungen an Natürlich müssen auch Hersteller di- 
den Nutzennachweis nicht besonders relevant zu sein.

Neben der Preiskomponente stellt aus unserer Sicht auch die Mengenkomponente ein Ausgabenrisiko dar. Im Gegensatz zu anderen verordneten Leistungen wie Arznei- oder Heilmitteln müssen sich die verordnenden Ärzte in keiner Art und Weise im Hinblick auf die Wirtschaftlichkeit der Verordnungen digitaler Gesundheitsanwendungen rechtfertigen. Die Verordnung einer erstattungsfähigen digitalen $\mathrm{Ge}$ sundheitsanwendung gilt automatisch als wirtschaftlich. Auch die Erstattung von digitalen Anwendungen durch die Krankenkassen ohne ärztliche Verordnung bei einer vorliegenden Indikation ist nicht unkritisch. Zwar haben Krankenkassen durchaus ein Interesse an wirtschaftlichen Erstattungsverfahren. Es ist aber in einem wettbewerblichen Krankenversicherungssystem durchaus üblich, dass Differenzierungsmöglichkeiten im Leistungsspektrum zur Risikoselektion genutzt werden (BVA 2018).

Ausgabenrisiken werden zudem durch die vorgesehene finanzielle Förderung digitaler Innovationen ausgelöst. Die Krankenkassen begrüßen zwar diese Möglichkeit, den Wettbewerb um die besten digitalen Versorgungsangebote anzuregen, sehen den Einsatz von Finanzmitteln der GKV aber auch als „Wagniskapital“ in einem Spannungsverhältnis zum Wirtschaftlichkeitsgrundsatz (AOK-Bundesverband 2019: 20). Mit der Regelung eröffnet der Gesetzgeber den Krankenkassen hier die Möglichkeit unternehmerischer Aktivitäten auf dem sogenannten zweiten Gesundheitsmarkt. Die Deutsche Gesellschaft für Gesundheitsökonomie merkt daher in ihrer Stellungnahme zum DVG zurecht an, dass dies aus europarechtlicher Sicht problematisch sein könnte (dggö 2019: 2).

\section{Fazit}

Vordergründig geht es beim DVG um eine Versorgungsoptimierung, denn digitale Anwendungen sollen durch das Umgehen der üblichen Verfahren im System der Selbstverwaltung schnellstmöglich in die Versorgung gebracht werden. Allerdings fehlt eine Einbettung in eine Gesamtstrategie der Digitalisierung des Gesundheitswesens: die wesentliche Voraussetzung für eine Vernetzung der
Akteure - die elektronische Patientenakte - wird durch das DVG nicht geschaffen. Ebenso wird eine Verknüpfung mit strategischen Gesundheitszielen nicht deutlich. Nicht zuletzt entsteht der Eindruck, dass digitale Gesundheitsanwendungen genau die Probleme im Bereich der Vernetzung und Integration der Akteure lösen könnten, die seit Jahren auf der Agenda stehen. Die WHO warnt vor dieser Überschätzung von digitalen Anwendungen: sie können Versorgungsstrukturen ergänzen und den Informationsaustausch beschleunigen, können allerdings nicht die klassischen Versorgungsstrukturen ersetzen (WHO 2019: XI).

Die Aushebelung der Selbstverwaltung mag die Einführung von Innovationen bei digitalen Gesundheitsanwendungen beschleunigen. Der Gesetzgeber schafft damit allerdings auch einen weiteren Akteur, der Einfluss auf die Gestaltung des Leistungskatalogs nehmen kann, so dass inhaltliche und wirtschaftliche Abstimmungsprozesse noch schwieriger werden. Damit wird die Integration von digitalen in die klassischen Versorgungsangebote eher erschwert als erleichtert.

Die im DVG nicht vorgesehene Nutzenbewertung oder gar Kosten-Nutzenbewertung für die digitalen Anwendungen wird mit einem Zirkelschluss begründet. Ziel des DVG ist es zwar, die Versorgungsqualität und Versorgungseffizienz zu verbessern. Die Zielerreichung wird allerdings tunlichst nicht gemessen, da digitale Anwendungen per se die Versorgung verbessern. Die WHO konstatiert allerdings nur wenig Evidenz über den Nutzen und noch weniger über die Effizienz von digitalen Anwendungen (WHO 2019: 86f.). Damit scheint das echte Interesse an der Verbesserung der Versorgung doch eher gering zu sein, insbesondere wenn die Informationen über diese potentiellen Verbesserungen durch den Hersteller der digitalen Gesundheitsanwendungen bereitgestellt werden.

Die Freiheiten, die das DVG bei der Finanzierung der Entwicklung von digitalen Gesundheitsanwendungen und der Preisgestaltung schafft, deuten vielmehr auf eine „Transformation von Gesundheitspolitik in Gesundheits- wirtschaftspolitik” (Egger 2019: 13). Krankenkassen dürfen durch das DVG Versichertengelder in Unternehmungen stecken. Auch hier wird wieder zirkulär argumentiert: die Förderung soll qua Gesetz zur Verbesserung von Qualität und Wirtschaftlichkeit beitragen - dabei ist gerade die Unsicherheit über das Ergebnis ein Merkmal von Unternehmungen. Damit werden Gelder, die die Versicherten für ihre Versorgungssicherheit aufwenden, in Projekte mit unsicherem Ausgang gesteckt. Nicht zuletzt das Risiko von Mondpreisen sowie das Risiko von Mengenausweitungen lassen befürchten, dass hier Chancen für Unternehmen geschaffen werden, ohne dass hinreichende Steuerungsmechanismen zum Schutz der Versichertengelder implementiert sind.

Die Digitalisierung und die Integration von Versorgungsstrukturen hängen eng zusammen. Bei ihrer Steuerung gibt es zahlreiche Parallelen. Bei der Digitalisierung versucht der Gesetzgeber über eine Zentralisierung der Steuerung und ein Umgehen der Selbstverwaltung die altbekannten Fehler bei der misslungenen 


\section{Literatur}

Amelung, V./P. Ex (2019). “Inkrementell oder mit der Brechstange: Wie wird das Gesundheitswesen endlich digital?" Gesundheits- und Sozialpolitik(1): 15-19.

Amelung, V. et al., Hrsg. (2017). Handbook Integrated Care, Springer International Publishing. AOK-Bundesverband (2019). Stellungnahme des AOK-Bundesverbandes zur Anhörung des Gesundheitsausschusses am 16.10.2019. Zum Entwurf eines Gesetzes für eine bessere Versorgung durch Digitalisierung und Innovation (Digitale-Versorgung-Gesetz DVG). Download unter https://www. aok-bv.de/imperia/md/aokbv/positionen/ stellungnahmen/aok_stellungnahme dvg.pdf am 15.11.2019.

Bertelsmann Stiftung (2018).

\#SmartHealthSystems.

Digitalisierungsstrategien im internationalen Vergleich, Download unter https://www. bertelsmann-stiftung.de/fileadmin/files/ Projekte/Der digitale Patient/VV SHSGesamtstudie_dt.pdf am 15.11.2019.
Bohm, S./S. Dudey (2019). “Zur Transmission erfolgreicher Innovationsfonds-Projekte in die GKV-Versorgung." Gesundheit und Gesellschaft Wissenschaft 19(3): 22-30.

BVA (2018). Sonderbericht zum Wettbewerb in der gesetzlichen Krankenversicherung. Bonn, Bundesversicherungsamt.

\section{Deutscher Bundestag (2019a).}

Beschlussempfehlung des Ausschusses

für Gesundheit zu dem Gesetzentwurf der Bundesregierung Entwurf eines Gesetzes für eine bessere Versorgung durch Digitalisierung und Innovation Digitale-Versorgung-Gesetz-

DVG. Berlin, Deutscher Bundestag, Drucksache 19/14867 vom 06.11.2019.

--- (2019b). Gesetzentwurf der

Bundesregierung. Entwurf eines Gesetzes für eine bessere Versorgung durch Digitalisierung und Innovation (Digitale-VersorgungGesetz - DVG). Berlin, Deutscher Bundestag, Drucksache 19/13438 vom 23.09.2019.

dggö (2019). Stellungnahme der dggö zum Entwurf eines Gesetzes für eine bessere
Versorgung durch Digitalisierung und Innovation (DVG). Download unter https://file. dggoe.de/presse/2019-06-07-Stellungnahme der_dggoe_zum_Digitale_Versorgung-Gesetz. pdf am 15.11.2019.

Egger, B. (2019). "Staatliche Eingriffe in den Leistungskatalog der GKV - Selbstverwaltung auf Abruf?" Gesundheits- und Sozialpolitik(4-5): 8-13.

Greß, S./S. Heinemann (2018). Germany: The Increasing Centralization of the Health Care Sector. Federalism and Decentralization in Health Care. G. P. Marchildon, T. J. Bossert. Toronto, Toronto University Press: 71-89.

Meister, S. (2019). “Das neue GesundheitsIch - souverän, selbstbestimmt und digital unterstützt?" Gesundheit und Gesellschaft Wissenschaft 19(3): 7-14

WHO (2019). Recommendations on digital interventions for health system strengthening Download unter https://www.who.int/ reproductivehealth/publications/digitalinterventions-health-system-strengthening/ en/ am 15. November 2019.

\section{Mit chronischen Erkrankungen umgehen}

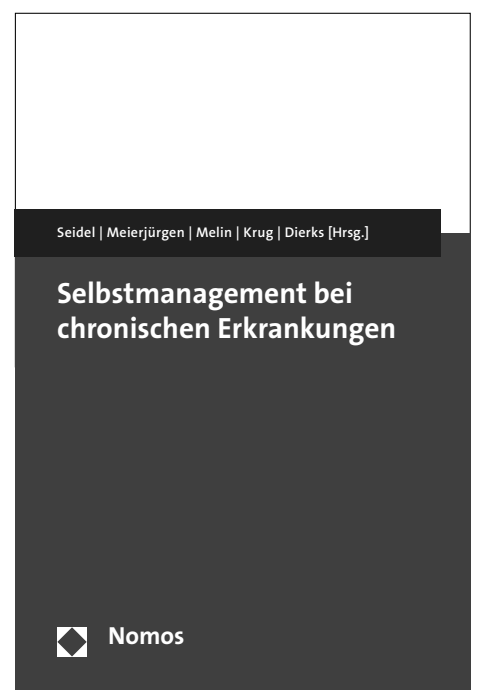

\section{Selbstmanagement bei chronischen Erkrankungen}

Herausgegeben von Dr. Gabriele Seidel, Dr. Rüdiger Meierjürgen, Susanne Melin, Jens Krug und Prof. Dr. Marie-Luise Dierks

2019, 309 S., brosch., 59,-€

ISBN 978-3-8487-4725-2

nomos-shop.de/37555

Selbstmanagementstrategien für Menschen mit chronischen Erkrankungen gewinnen in der Medizin an Bedeutung. Der Sammelband gibt einen Überblick über die aktuelle Diskussion, beschreibt die Umsetzung des INSEA-Programms in Deutschland und stellt internationale und nationale Programme zur Förderung des Selbstmanagements für chronisch kranke Menschen vor.

\footnotetext{
베IIII Nomos

点 e Library www.nomos-elibrary.de
}

Bestellen Sie im Buchhandel oder versandkostenfrei online unter nomos-shop.de Bestell-Hotline (+49)7221.2104-37 | E-Mail bestellung@nomos.de | Fax (+49)7221.2104-43 Alle Preise inkl. Mehrwertsteuer 